\title{
The soft X-ray spectrum of PG1211+143
}

\author{
K. A. Pounds, ${ }^{1}$ K. L. Page ${ }^{1}$ and J. N. Reeves ${ }^{2}$ \\ ${ }^{1}$ Department of Physics and Astronomy, University of Leicester, UK \\ ${ }^{2}$ NASA Goddard Space Flight Center, Code 663, Greenbelt, MD 20771, USA
}

\begin{abstract}
The narrow line QSO PG1211+143 has been a focus of recent attempts to understand the soft excess in AGN, while the 2001 XMM-Newton observation of this luminous AGN also provided evidence for a massive and energetic outflow. Here we consider a physical link between the energetic outflow and the variable soft excess.
\end{abstract}

Keywords. Galaxies: active - galaxies: nuclei

\section{Introduction}

One of the most important new results emerging from XMM-Newton and Chandra observations of AGN is the evidence for sub-relativistic outflows from a number of luminous quasars. For two of these, APM08279+5255 (Chartas et al. 2002) and PG1115+080 (Chartas et al. 2003), both BAL quasars, the immediate inference was to associate the X-ray columns with the high velocity gas seen in optical and UV spectra of these unusual objects. In such cases the high columns might result from viewing the continuum source through a wind being blown tangentially off the accretion disc (Proga et al. 2000).

However, in 2 further cases, PG1211+143 (Pounds et al. 2003) and PDS456 (Reeves et al. 2003), a moderate and a high luminosity QSO, respectively, evidence of a high velocity - and hence energetic - outflow suggested this could be a common property of luminous, or perhaps more specifically, of high accretion rate quasars (King and Pounds 2003). Unless highly collimated, such outflows will form a substantial component of the mass and energy budgets in luminous AGN, while having important implications for metal enrichment of the intergalactic medium and for the feedback mechanism implied by the correlation of black hole and galactic bulge masses (King 2005).

The XMM-Newton observation of PG1211+143 in 2001 has also been used recently to explore the physical origin of the 'soft excess' in the X-ray spectra of many AGN, following doubts raised about Comptonisation models (Gierlinski and Done 2004). Alternative descriptions have been proposed, invoking strong reflection from a highly ionised accretion disc (Crummy et al. 2006) and absorption in high velocity ionised gas (Schurch and Done 2006, Chevallier et al. 2006). Here we also examine EPIC data from a second observation of PG1211+143 in 2004, where a conventional plot shows a weaker but 'hotter' soft excess (Figure 1L).

A direct comparison of the EPIC data from 2001 and 2004 (Figure 1R) suggests the spectral difference is primarily due to a variable 'deficit' of flux at $\sim 0.7-2 \mathrm{keV}$, supporting the contention that the spectral curvature near $\sim 1 \mathrm{keV}$ is indeed an artefact of absorption on a steeper underlying continuum. However, if the flux deficit is due to photoionised absorption, it is interesting that the hard X-ray flux - which should be a good proxy for the ionising luminosity - is essentially unchanged, suggesting that a reduced covering factor (or column density) is the cause of the weaker 'soft excess' in 2004. Here we suggest an alternative possibility (explored more fully in Pounds and Reeves 2006), that a variable continuum component partly 'fills in' the absorption flux deficit in the 2004 

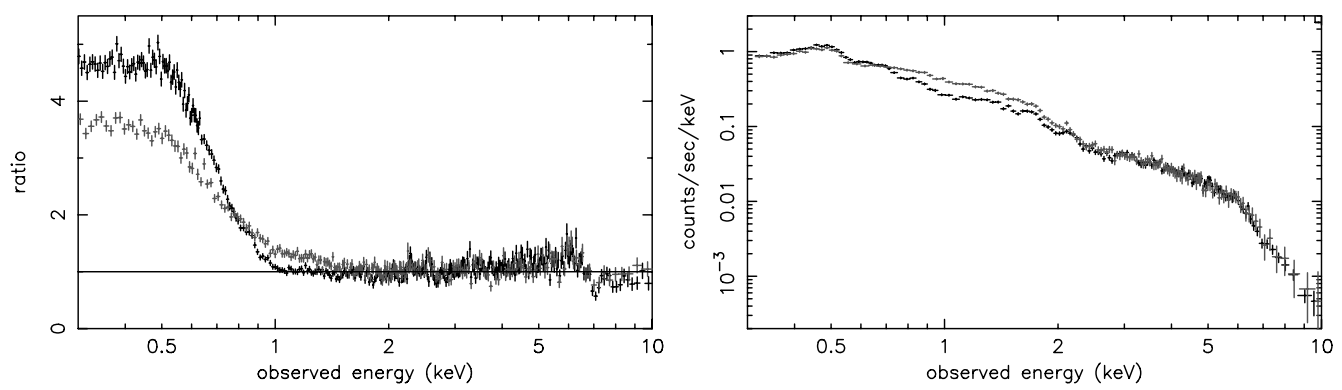

Figure 1. Left panel: conventional plot of the 2001 (black) and 2004 (grey) EPIC pn spectra showing a strong soft excess sitting above the hard power law. On this view it appears that the soft excess in 2004 is weaker but 'hotter'. Right panel: direct comparison of the backgroundsubtracted EPIC data showing the spectral difference to be due to an increase in flux at $\sim 0.7-2$ $\mathrm{keV}$ and a (less obvious, but significant) decrease at $\sim 0.4-0.7 \mathrm{keV}$ in 2004 (grey).

spectrum. While contributing directly to the strong soft X-ray flux in PG1211+143, the additional soft continuum component also explains the lack of strong 'narrow' absorption lines in the soft X-ray band (Kaspi and Behar 2006), in conflict with the relatively strong, blue-shifted absorption lines in the EPIC data (Pounds and Page 2006).

We speculate that the additional, steep power law continuum is powered by the energetic high velocity outflow.

\section{Confirming an energetic outflow in PG1211+143}

PG1211+143 (at $z=0.0809$ ) is a prototypical 'Big Blue Bump' quasar, and one of the brightest AGN in the soft X-ray band (Elvis et al. 1991). It is also classified (Kaspi et al. 2000) as a Narrow Line Seyfert 1 galaxy (FWHM H $\beta 1800 \mathrm{~km} \mathrm{~s}^{-1}$ ), with a small central black hole mass $\left(\sim 4 \times 10^{7} M_{\odot}\right)$ for an object of bolometric luminosity $4 \times 10^{45} \mathrm{erg} \mathrm{s}^{-1}$, implying that the accretion rate is close to the Eddington limit.

The initial $\sim 50$ ks observation of PG1211+143 with XMM-Newton in 2001 revealed several blue-shifted absorption lines, interpreted by Pounds et al. (2003) as evidence for a highly ionised outflow with a velocity $\mathrm{v} \sim 0.09 c$, a conclusion primarily based on identifying the strongest $\sim 7 \mathrm{keV}$ feature with FeXXVI Ly $\alpha$. As Kaspi and Behar (2006) were unable to confirm this result in a careful ion-by-ion modelling of the RGS data we have re-examined the higher signal-to-noise EPIC data. That re-analysis resolved additional absorption lines in the intermediate $(\sim 1-4 \mathrm{keV})$ energy band (Pounds and Page 2006), strengthening the evidence for a high velocity outflow. In total, 7 statistically significant absorption lines are identified, with the strong $\sim 7 \mathrm{keV}$ line re-assigned to He $\alpha$ of FeXXV, yielding an increased outflow velocity $\mathrm{v} \sim 0.14 \pm 0.01 c$.

Assuming a spherically symmetric flow, the mass loss rate is of then of order $\dot{M} \sim$ $35 b M_{\odot} \mathrm{yr}^{-1}$, where $b \leqslant 1$ allows for the collimation of the flow. Modelling of the broadband spectrum of PG1211+143 (Pounds and Reeves 2006) has quantified both the absorbed and re-emitted fluxes for the ionised outflow, yielding a covering factor $\mathrm{CF} \sim 0.1$ for the high velocity, highly ionised outflow, and $\dot{M} \sim 3.5 M_{\odot} \mathrm{yr}^{-1}$. This outflow rate compares with $\dot{M}_{\text {Edd }}=1.6 M_{\odot} \mathrm{yr}^{-1}$ for a non-rotating $\mathrm{SMBH}$ of mass $\sim 4 \times 10^{7} M_{\odot}$ (Kaspi et al. 2000) accreting at an efficiency of $10 \%$. The mechanical energy of the highly ionised outflow is then $\sim 10^{45} \mathrm{erg} \mathrm{s}^{-1}$, easily sufficient to power a substantial component of the $\mathrm{X}$-ray emission of PG1211+143. 

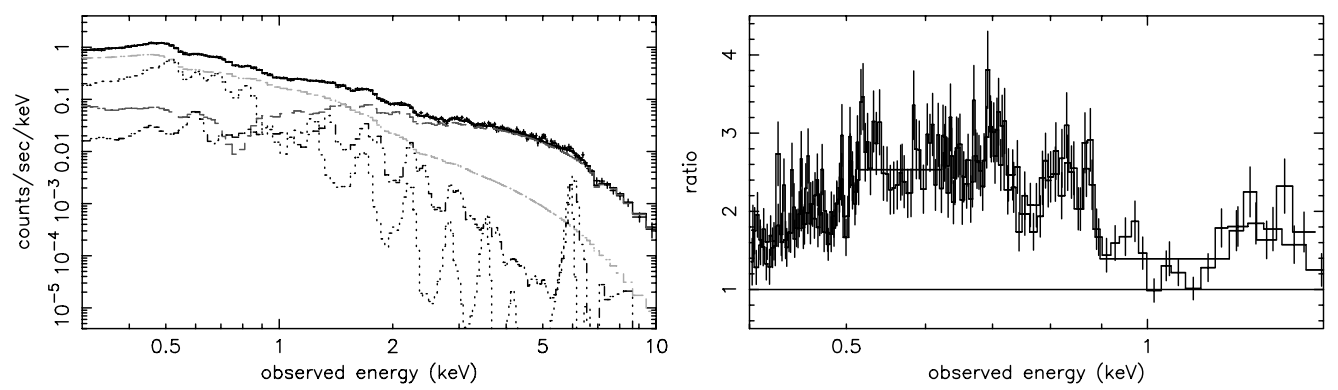

Figure 2. Left panel: deconvolution of the EPIC MOS spectrum of PG1211+143 with the primary (dark grey) and secondary (light grey) continua plus line emission from the highly and moderately ionised outflow (Pounds and Reeves 2006). Right panel: structure in the soft excess in the 2001 RGS data. XSTAR model fitting shows this structure is dominated by velocity-broadened resonance emission lines of $\mathrm{N}, \mathrm{O}, \mathrm{Ne}$ and $\mathrm{Mg}$, together with a group of Fe-L lines at $\sim 0.7 \mathrm{keV}$.

\section{A second continuum component}

For most AGN the energetically dominant emission component can be modelled by a power law of photon index $\Gamma \sim 1.9$ (Nandra and Pounds 1994), visible in Type 1 objects over the $\sim 2-100 \mathrm{keV}$ band. This 'primary' continuum component is widely believed to arise by Comptonisation of UV and optical accretion disc photons in a high temperature electron corona (Haardt and Maraschi 1991). However, recent studies of spectral variability in MCG-6-30-15 (Vaughan and Fabian 2004) and 1H0419-57 (Pounds et al. 2004) have provided clear evidence of a variable power law component of slope significantly steeper than the 'canonical value' of $\Gamma \sim 1.9$.

We now suggest this softer continuum component is physically distinct from the 'primary' continuum in PG1211+143, having established that the mechanical energy in the fast, highly ionised outflow is ample to power that additional continuum component, perhaps via internal shocks (analogous to the process suggested in Gamma Ray Bursts), or shock heating of slower moving clouds providing the bulk of the continuum opacity. The most likely X-ray emission mechanism would again be Comptonisation of optical-UV disc photons, with the steep power law resulting from a lower equilibrium temperature in the dispersed 'corona' compared with that responsible for the primary power law continuum.

A separate continuum component is attractive in the present context since, if absorption is to create the impression of a strong 'soft excess', then the underlying continuum must be steeper than observed above $\sim 2 \mathrm{keV}$.

\section{A new spectral model}

The evidence of absorption in highly ionised (narrow absorption lines) and moderately ionised gas (low energy spectral curvature) suggests a new spectral model including 2 power law continua, both modified by absorption, and re-emission from a 2-component photoionised gas. Applying this model to the 2001 data from PG1211+143 produced a statistically excellent fit (Pounds and Reeves 2006).

Figure 2L illustrates the model components, showing that above $\sim 2 \mathrm{keV}$ the spectrum is dominated by the 'primary' power law, with a photon index $\Gamma \sim 2.2$, at the upper end of - but consistent with - the 'accepted' range for type 1 AGN spectra. At lower energies the secondary power law component $(\Gamma \sim 3.1)$ dominates, while below $\sim 1 \mathrm{keV}$ re-emission, particularly from the moderately ionised absorber, becomes significant. 
Strong absorption of the primary power law is seen to be responsible for the mid-band $(\sim 2-6 \mathrm{keV})$ spectral curvature, with ionised gas column densities of $N_{H} \sim 3-5 \times 10^{22}$ $\mathrm{cm}^{-2}$. In both pn and MOS spectral fits the model shows a broad trough near $\sim 0.75-$ $0.8 \mathrm{keV}$, due to Fe-L absorption. Of particular relevance in the present context, the luminosity of the steep, less absorbed, power law component was $\sim 6 \times 10^{43} \mathrm{erg} \mathrm{s}^{-1}$, more than an order of magnitude lower than the estimated fast outflow energy.

In modelling spectral structure in the higher resolution RGS data (Figure 2R), photoionised line emission had to be convolved with a gaussian $(\sigma \sim 25 \mathrm{eV}$ at $0.6 \mathrm{keV})$, corresponding to velocity broadening of $29000 \mathrm{~km} \mathrm{~s}^{-1}$ (FWHM), consistent with the high velocity flow.

Applying the 2001 model to the 2004 data, a good fit was obtained with element abundances and ionisation parameters fixed, the spectral change being largely modelled by an increase in the steep, weakly absorbed continuum component. In contrast, a change in the covering factor (modelled by a lower column) of ionised absorber on the primary power law was not able - alone - to fit both data sets.

\section{Broader application of the new spectral model}

The Black Hole Winds model (King and Pounds 2003) provides a simple physical basis whereby massive, high velocity outflows can be expected in AGN accreting at or above the Eddington limit. In addition to PG1211+143, this might apply to both PDS456 (Reeves et al. 2003) and to the bright Seyfert 1 IC4329A, recently shown (Markowitz et al. 2006) to exhibit a strongly blue-shifted Fe K absorption line indicating a highly ionised outflow at $\mathrm{v} \sim 0.1 c$. While relatively rare in the local universe such $\mathrm{X}$-ray spectra could be common for luminous, higher redshift AGN.

If the outflow velocity in PG1211+143 equates to the escape velocity at the launch radius $R_{\text {launch }}$ (from an optically thick photosphere or radiatively extended inner disc), $\mathrm{v} \sim 0.14 c$ corresponds to $R_{\text {launch }} \sim 50 R_{\mathrm{s}}$ (where $R_{\mathrm{s}}=2 G M / c^{2}$ is the Schwarzschild radius), or $3 \times 10^{14} \mathrm{~cm}$ for PG1211+143. The EPIC data show significant flux variability in the harder (2-10 keV) band on timescales of 2-3 hours (fig 1 in Pounds et al. 2003)), compatible with the above scale size relating to the primary (disc/corona) X-ray emission region.

Observations of PG1211+143 over several days are needed to test the new spectral model. The variability timescale of the secondary power law will constrain the scale of the region where we predict the fast outflow undergoes internal shocks. Detecting hard X-ray emission from PG1211+143 above $\sim 20 \mathrm{keV}$ would also support the need for a continuum component with photon index less steep than that in the single power law/ absorption models, while deeper RGS spectra are needed to resolve the broad emission line profiles indicated in the model fits.

In summary, it seems clear that further studies of high accretion rate AGN, such as PG1211+143, offer great potential for understanding outflows and their potential effects on the intergalactic medium and host galaxy growth.

\section{References}

Chartas G., Brandt W. N., Gallagher S. C. \& Garmire G. P. 2002, ApJ, 579, 169

Chartas G., Brandt W. N. \& Gallagher S. C. 2003, ApJ, 595, 85

Chevallier L., Collin S., Dumont A-M., Czerny B., Mouchet M., Goncalves A. C. \& Goosmann R. 2006, A\&A, 449, 493

Crummy J., Fabian A. C., Gallo L. \& Ross R. R. 2006, MNRAS, 365, 1067 
Elvis M., Giommi P., Wilkes B. J. \& McDowell J. 1991, ApJ, 378, 537

Gierlinski M. \& Done C. 2004, MNRAS, 349, L7

Haardt F. \& Maraschi L. 1991, ApJ, 350, L81

Kaspi S., Smith P. S., Netzer H., Maoz D., Jannuzi B. T. \& Giveon U. 2000, ApJ, 533, 631

Kaspi S. \& Behar E. 2006, ApJ, 636, 674

King A. R. \& Pounds K. A. 2003, MNRAS, 345, 657

King A. R. 2005, ApJ, 635, L121

Markowitz A., Reeves J. N. \& Braito V. 2006, ApJ, 646, 783

Nandra K. \& Pounds K. A. 1994, MNRAS, 268, 405

Pounds K. A., Reeves J. N., King A. R., Page K. L., O'Brien P. T. \& Turner M. J. L. 2003, MNRAS, 345, 705

Pounds K. A., Reeves J. N., Page K. L. \& O'Brien P. T. 2004, ApJ, 616, 696

Pounds K. A. \& Page K. L. 2006, MNRAS, 372, 1275

Pounds K. A. \& Reeves J. N. 2006, MNRAS, accepted (astro-ph/0610112)

Proga D., Stone J. M. \& Kallman T. R. 2000, ApJ, 543, 686

Reeves J. N., O'Brien P. T. \& Ward M. J. 2003, ApJ, 593, L65

Schurch N. J. \& Done C. 2006, MNRAS, 371, 81

Vaughan S. \& Fabian A. C. 2004, MNRAS, 348, 1415

Kazuo Makishima: Comment: Steep power law you are finding is extremely interesting and reminds me of similar phenomenon that occurs in Galactic black hole binaries when they get in the very high state. In that case this component appears between $10-50 \mathrm{keV}$, connecting the hard tail and the soft disc component.

Giorgio MatT: Can you exclude that the $0.7-2 \mathrm{keV}$ spectrum is due to ionized reflection rather than absorption?

Kenneth Pounds: The spectral curvature (soft excess) in PG1211 is too strong to be produced by photoionized reflection from the accretion disc unless the direct continuum is somehow suppressed, for example by light bending in strong gravity near the black hole. Given the detection of a large column density outflow in this luminous AGN, absorption plus re-emission provides a more likely explanation. 


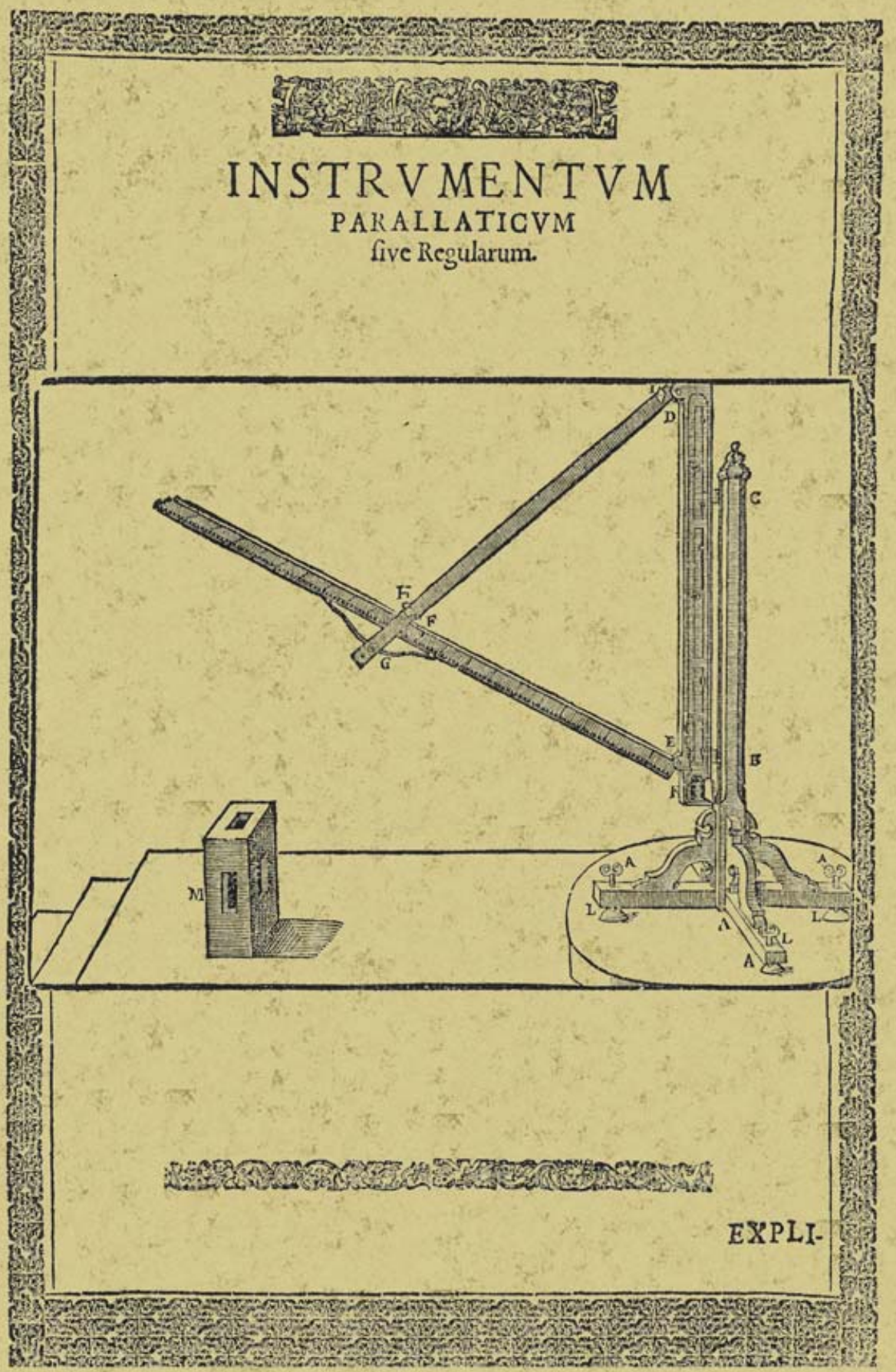

